\title{
An Efficient Test Pattern Selection Method for Improving Defect Coverage with Reduced Test Data Volume and Test Application Time
}

\author{
Zhanglei Wang and Krishnendu Chakrabarty \\ Department of Electrical and Computer Engineering \\ Duke University, Durham, NC 27708, USA \\ Email: \{zw8,krish\}@ee.duke.edu
}

\begin{abstract}
Testing using $n$-detection test sets, in which a fault is detected by $n(n>1)$ input patterns, is being increasingly advocated to increase defect coverage. However, the data volume for an $n$-detection test set is often too large, resulting in high testing time and tester memory requirements. Test set selection is necessary to ensure that the most effective patterns are chosen from large test sets in a high-volume production testing environment. Test selection is also useful in a timeconstrained wafer-sort environment. We use a probabilistic fault model and the theory of output deviations for test set selection-the metric of output deviation is used to rank candidate test patterns without resorting to fault grading. To demonstrate the quality of the selected patterns, we present experimental results for resistive bridging faults and nonfeedback zero-resistance bridging faults in the ISCAS benchmark circuits. Our results show that for the same test length, patterns selected on the basis of output deviations are more effective than patterns selected using several other methods.
\end{abstract}

\section{INTRODUCTION}

Shrinking process technologies and the use of new materials such as copper interconnects and high-K dielectrics are leading to increasingly complex defects for integrated circuits (ICs) [1], [2]. It is not always possible to accurately model these defects or predict their occurrences. A single spot defect is likely to affect several logic gates and multiple defects on a die are likely. The locations of these defects are unpredictable. The failure probability of a gate due to a defect depends also on factors such as die temperature and power supply variations. As a result, it is no longer adequate to rely only a deterministic fault model, especially a fault model that targets only single faults. In addition, many real defects are difficult to model by existing fault models; examples of such defects include resistive opens and shorts, crosstalk, and interconnect defects. Thus while classical fault models are widely used in industry [3]-[6], it is also important to focus on alternative methods to catch defects that are not fully understood.

It has been shown in the literature that $n$-detection test sets, where each stuck-at fault is targeted by $n(n>1)$ different patterns, are effective for detecting unmodeled defects [7]-[10]. As the number of unique detections for each fault increases, the defect coverage usually improves compared with other test generation methods. An advantage of this approach is that even when $n$ is very large, $n$-detection test sets can be generated using existing single stuck-at ATPG tools with reasonable computation time.

However, the data volume of an $n$-detection test set is often too large, resulting in long testing time and high tester memory requirement [11], [12]. This is because the $n$-detection method simply tries to detect each single stuck-at fault $n$ times, and does not use any other metric to evaluate the contribution of a test pattern towards increasing the defect coverage. It has been reported in the literature that the sizes of $n$-detection test sets tend to grow linearly with $n$ [12]. Therefore, test selection is necessary to ensure that the most effective test patterns are chosen from large test sets during timeconstrained and high-volume production testing [13]. Due to test time constraints, it is often not possible to apply all the $n$-detection patterns, even for relatively small values of $n$ [14]. Test selection is also useful in a time-constrained wafer-sort environment. Even if test data volume and test application time are reduced using test compression, test pattern selection can still be used to reorder a test set. If highly effective test patterns are applied first, a defective chip can fail earlier, further reducing test application time in an abort-atfirst-fail environment. Moreover, test compression is not effective if the patterns are delivered without a significant fraction of don't-care bits. In such cases, test set selection can be a practical method to reduce test time and test data volume.

The test set selection problem can be addressed using a probabilistic fault model, recently published in [15], that allows any number of gates in the IC to fail probabilistically. Tests for this fault model, determined using the theory of output deviations, can be used to supplement tests for classical fault models, thereby increasing test quality and reducing the probability of test escape. In this paper, we use the output deviation metric for test selection. We also indirectly generate an $n$-detection test set by selecting efficient patterns based on output deviation from a larger $n^{\prime}$-detection test set $\left(n^{\prime}>n\right)$. To evaluate the quality of the selected test patterns, we determine the coverage that they provide for resistive bridging faults [16] and single non-feedback zero-resistance bridging faults (s-NFBFs). Experimental results show that patterns selected using the probabilistic fault model and output deviations provide higher bridging fault coverage than patterns selected using other methods.

We also use metrics for gate exhaustive (GE) testing [17] to evaluate the effectiveness of deviation-based test sets. In a recent production test experiment, more than 50,000 chips were analyzed to understand the correlation between the number of defective chips screened by a set of test patterns and the coverage provided by these test patterns with respect to various test metrics [18]. The GE metric was found to have higher correlation compared to the SSL and the bridge coverage estimate metrics. Therefore we also consider GE metrics to evaluate deviation-based test sets. Our experiments show that deviation-based test sets outperform $n$-detect test sets for several GE metrics.

The remainder of this paper is organized as follows. Sections II introduces the probabilistic fault model and the theory of output deviations. The design of experiments and some experimental results are reported in Section IV. Section V concludes this paper and outlines directions for future work.

\section{THE PROBABILISTIC FAULT MODEL AND THE THEORY OF OUTPUT DEVIATIONS}

The confidence level of a single-output gate encompasses all the different input combinations of the gate, and for a given innut combination, it provides the probability that the gate output 


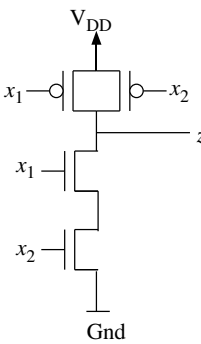

(a)
$(0.90 .90 .90 .8)$

(b)

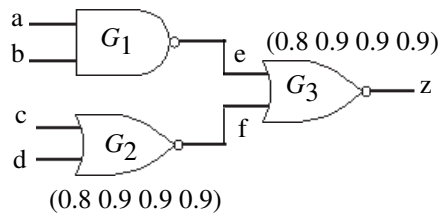

Fig. 1. 2-input NAND gate and an illustrative example.

for the corresponding input combination. The probability that a gate output is correct can be different for the various input combinations.

Definition 1: The confidence level $R_{i}$ of a gate $G_{i}$ with $m$ inputs and a single output is a vector with $2^{m}$ components, defined as: $R_{i}=$ $\left(r_{i}^{(00 \ldots 00)} r_{i}^{(00 \ldots 01)} r_{i}^{(00 \ldots 10)} \ldots r_{i}^{(11 \ldots 11)}\right)$, where each component of $R_{i}$ denotes the probability that the gate output is correct for the corresponding input combination.

For example, $r_{i}^{00}$ is the probability that the output of a 2-input $G_{i}$ is correct under input 00 . If $m=2$ for a logic gate, we have $R_{i}=\left(r_{i}^{(00)} r_{i}^{(01)} r_{i}^{(10)} r_{i}^{(11)}\right)$.

The above gate-level confidence level vectors can be generated in a number of ways, e.g., using layout information, inductive fault analysis [19], and failure data analysis. It can also be estimated using simple transistor-level failure probabilities. Our objective here is not to develop new techniques for determining confidence level vectors, but rather to use these as inputs for the computation of output deviation. In practice, deviation-based test patterns can be generated using multiple sets of confidence level vector estimates.

Consider the 2-input NAND gate shown in Fig. 1(a). Suppose each transistor can be stuck-open due to a defect, i.e., it cannot be switched on, with probability $\alpha$. Similarly, suppose each transistor can be stuck-on due to a defect, i.e., it cannot be switched off, with probability $\beta$. Next let us consider input combination $x_{1} x_{2}=00$. If only stuck-open faults are considered, the NAND gate produces the correct output for this combination with probability $r^{(00)}=(1-\alpha)^{2}$ because the gate produces an incorrect output only if both transistors are stuck-open. Likewise, if we only consider stuck-on faults, the probability that the NAND gate produces the correct output for input 00 is $r^{(00)}=(1-\beta)^{2}$.

Next we associate signal probabilities $p_{i, 0}$ and $p_{i, 1}$ with each line $i$ in the circuit, where $p_{i, 0}$ and $p_{i, 1}$ are the probabilities for line $i$ to be at logic 0 and 1 , respectively. Obviously we have $p_{i, 0}+p_{i, 1}=1$. The calculation of the signal probabilities is along the same lines as introduced in [20], and used later in [21]. To reduce the amount of computation as in [20], [21], signal correlations due to reconvergent fanout are not considered here.

Let $i$ be the output of a two-input gate $G$. Let $j$ and $k$ denote the input lines for this gate. If $G$ is a NAND gate, we have:

$$
\begin{aligned}
p_{i, 0} & =p_{j, 1} p_{k, 1} r_{i}^{(11)}+p_{j, 0} p_{k, 0}\left(1-r_{i}^{(00)}\right) \\
& +p_{j, 0} p_{k, 1}\left(1-r_{i}^{(01)}\right)+p_{j, 1} p_{k, 0}\left(1-r_{i}^{(10)}\right) \\
p_{i, 1} & =p_{j, 0} p_{k, 0} r_{i}^{(00)}+p_{j, 0} p_{k, 1} r_{i}^{(01)} \\
& +p_{j, 1} p_{k, 0} r_{i}^{(10)}+p_{j, 1} p_{k, 1}\left(1-r_{i}^{(11)}\right)
\end{aligned}
$$

Likewise, the signal probabilities can be easily computed for other gate types.

Figure 1(b) shows a simple circuit consisting of three gates $G_{1}$, $G_{2}$, and $G_{3}$ with confidence level vectors (0.9 0.90 .90 .8$)$, ( 0.8

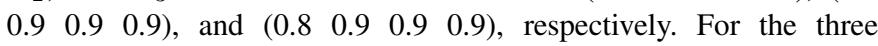
different deterministic input vectors 0000,0101 , and 1111, the signal

\begin{tabular}{||c|c|c|c|c|c|c||}
\hline Input pattern, $z$ & $p_{e, 0}$ & $p_{e, 1}$ & $p_{f, 0}$ & $p_{f, 1}$ & $p_{z, 0}$ & $p_{z, 1}$ \\
\hline 0000,0 & 0.1 & 0.9 & 0.2 & 0.8 & 0.886 & 0.114 \\
0101,0 & 0.1 & 0.9 & 0.9 & 0.1 & 0.837 & 0.163 \\
1111,1 & 0.8 & 0.2 & 0.9 & 0.1 & 0.396 & 0.604 \\
\hline
\end{tabular}

TABLE I

SIGNAL PROBABILITIES FOR DIFFERENT INPUT COMBINATIONS

probabilities are determined and presented in Table I. The fault-free values at the output $z$ are also listed in the first column of Table I.

In [22], probabilistic transfer matrices (PTMs), which are similar to confidence level vectors, have been used for generating test patterns for soft errors caused by single-event upsets. The focus of this work however is on test pattern selection for permanent faults. Signal correlation due to reconvergent fanouts is accounted for in [22] using tensor products, hence PTMs may not scale well for large circuits.

We next use the concept of signal probabilities to develop the notion of output deviations. We then describe how to determine deviation-based test patterns that can be used to enrich test sets.

For any logic gate (or primary output) $g$ in a circuit, let its faultfree output value for any given input pattern $t_{j}$ be $d, d \in\{0,1\}$. The output deviation $\Delta_{g, j}$ of $g$ for input pattern $t_{j}$ is defined as $p_{g, \bar{d}}$, where $\bar{d}$ is the complement of $d$. Intuitively, the deviation for an input pattern is a measure of the likelihood that the gate output is incorrect for that input pattern.

Next we formally define the probabilistic fault model for a combinational circuit $\mathcal{C}$.

Definition 2: A combinational circuit $\mathcal{C}$ is defined as $\mathcal{C}=$ $\{\mathcal{G}, \mathcal{P} \mathcal{I}, \mathcal{Z}, \mathcal{R}\}$, where $\mathcal{G}=\left\{G_{1}, G_{2}, \ldots, G_{N}\right\}$ is the set of logic gates in $\mathcal{C}, \mathcal{P} \mathcal{I}$ and $\mathcal{Z}$ are the sets of primary inputs and outputs respectively, and $\mathcal{R}=\left\{R_{1}, R_{2}, \ldots, R_{N}\right\}$ is the set of confidence level vectors of the gates in $\mathcal{G}$.

Definition 3: A probabilistic fault model $\mathcal{F}$ for circuit $\mathcal{C}$ is defined as follows: (i) Each gate $G_{i}$ can fail independently of other gates; (ii) the fault behavior of $\mathcal{C}$ is defined by $R_{i}$.

Under this fault model, the expected output values of the circuit in response to an input pattern is no longer deterministic. Rather, it is given by the signal probabilities at primary outputs. Note that the circuit behavior is assumed to be deterministic after manufacturing; the probabilistic fault model is only used during test development for pattern grading.

The probability that $t_{j}$ will produce an observable error at $z_{i}$ for fault model $\mathcal{F}$ is directly proportional to $\Delta_{z_{i}, j}$. The goal of testing is to apply those vectors to $\mathcal{C}$ that produce large deviations at the output. Therefore, the concept of output deviations offers a promising method for modeling pattern quality. From now on, we only consider output deviations at outputs and use the terms "output deviation" and "deviation" interchangeably.

\section{TEST PATTERN SELECTION}

In this section, we attempt to select a relatively small number of test patterns, using the output deviations, from a repository test set $T_{r e p}$ that contains a much larger number of test patterns. The selected test set should provide high defect coverage.

We choose to use an $n_{0}$-detection test set as $T_{\text {rep }}\left(n_{0}=15\right.$ for smaller benchmark circuits and $n_{0}=7$ for larger benchmark circuits). To generate $T_{r e p}$, we first run Atalanta [23], a single stuckat fault ATPG tool, to generate $n_{0}$ test patterns for each single stuck-at fault. (It is not guaranteed that Atalanta can generate $n_{0}$ patterns for each fault, but instances when it fails to do so are rare.) Since these test patterns contain unspecified bits (Xs), they are further compacted and randomly-filled to form $T_{\text {rep }}$.

For comparison purposes, we also generate a group of $n$-detection test sets $(n=2,3 \ldots 8)$ as baseline test sets by randomly 


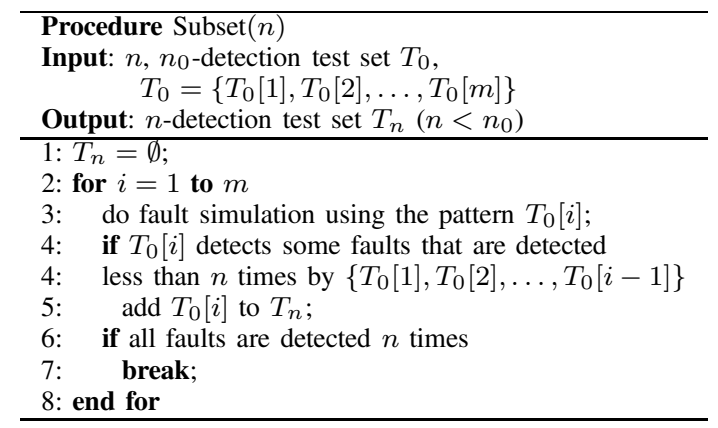

Fig. 2. Procedure to generate an $n$-detection test set from a $n_{0}$-detection test set $\left(n<n_{0}\right)$.

\begin{tabular}{|c|c|c|c|}
\hline \multirow{2}{*}{$\begin{array}{c}\text { Pattern } \\
\text { ID }\end{array}$} & \multicolumn{3}{|c|}{ Output deviations } \\
\cline { 2 - 4 } & Port A & Port B & Port C \\
\hline $\mathrm{t} 1$ & 0.9 & 0.2 & 0.7 \\
\hline $\mathrm{t} 2$ & 0.8 & 0.3 & 0.4 \\
\hline $\mathrm{t} 3$ & 0.6 & 0.7 & 0.8 \\
\hline $\mathrm{t} 4$ & 0.2 & 0.6 & 0.5 \\
\hline $\mathrm{t} 5$ & 0.7 & 0.4 & 0.9 \\
\hline $\mathrm{t} 6$ & 0.3 & 0.1 & 0.2 \\
\hline
\end{tabular}

(a) Test patterns and output deviations.

\begin{tabular}{|c|c|c|c|c|c|c|}
\hline Port A & $\mathrm{t} 1$ & $\mathrm{t} 2$ & $\mathrm{t} 5$ & $\mathrm{t} 3$ & $\mathrm{t} 6$ & $\mathrm{t} 4$ \\
\hline Port B & $\mathrm{t} 3$ & $\mathrm{t} 4$ & $\mathrm{t} 5$ & $\mathrm{t} 2$ & $\mathrm{t} 1$ & $\mathrm{t} 6$ \\
\hline Port C & $\mathrm{t} 5$ & $\mathrm{t} 3$ & $\mathrm{t} 1$ & $\mathrm{t} 4$ & $\mathrm{t} 2$ & $\mathrm{t} 6$ \\
\hline
\end{tabular}

(b) Outcome of the sorting procedure (Matrix $X$ ).

Fig. 3. An example to illustrate the sorting of $T_{r e p}$ based on output deviations.

test patterns from $T_{r e p}$. Each time a test pattern is selected, we perform fault simulation and drop those faults that are already detected $n$ times. This procedure, as outlined in Fig. 2, continues until all faults are detected $n$ times. The set $T_{r e p}$ is randomly reordered before being provided as input to Procedure Subset. Therefore, although this procedure goes through $T_{0}$ sequentially (line 2), it tends to select patterns from $T_{0}$ randomly. The test sets selected using output deviations are compared to these $n$-detection test sets in terms of their sizes and defect coverage.

Next, we sort $T_{r e p}$ such that test patterns with high deviations can be selected earlier than test patterns with low deviations. For each primary output (PO), all test patterns in $T_{r e p}$ are sorted in descending order based on their output deviations. The result is stored in a matrix $X$ with $M$ rows and $P$ columns, where $M$ is the number of POs and $P$ is the number of test patterns in $T_{r e p}$. The element in the $i$-th row and the $j$-th column is the test pattern that has the $j$-th highest output deviation at the $i$-th PO. Fig. 3 illustrates this sorting procedure. From matrix $X$, a new ordered test set $T_{r e p}^{\prime}$ can be obtained using Procedure Deviation_Sort shown in Fig. 4. For the example in Fig. 3 , the resulting sorted test set is $T_{r e p}^{\prime}=\left\{t_{1}, t_{3}, t_{5}, t_{2}, t_{4}, t_{6}\right\}$.

We next describe three different ways to select a small number of

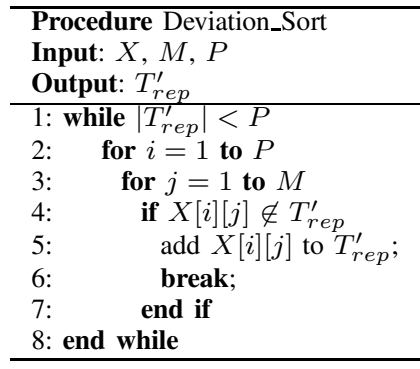

Fig. 4. Procedure to select patterns with high deviations.

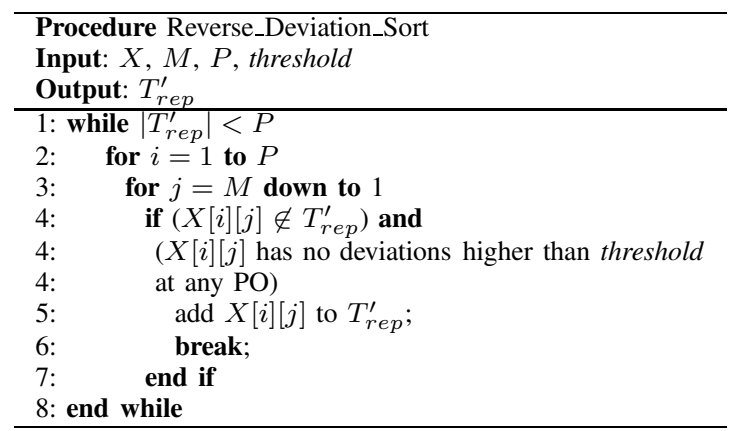

Fig. 5. Procedure to reverse_sort test patterns on the basis of deviations.

patterns from $T_{r e p}^{\prime}$, such that the resulting test sets can be compared with the baseline $n$-detection test sets.

Method 1: Generate an $n$-detection test set using Procedure Subset shown in Fig. 2. However, unlike the baseline $n$-detection test sets, the input to Procedure Subset is $T_{r e p}^{\prime}$ instead of $T_{r e p}$, such that test patterns with high deviations are selected first. Therefore, the resulting test set is an optimized $n$-detection test set that usually contains a smaller number of patterns and achieves a higher defect coverage than its baseline $n$-detection test set.

Method 2: Select patterns with high output deviations from $T_{r e p}^{\prime}$. The test patterns in $T_{r e p}^{\prime}$ are selected sequentially to form the resulting test set.

Method 3: Select patterns with low output deviations from $T_{r e p}^{\prime}$ using Procedure Reverse_Deviation_Sort shown in Fig. 5. Procedure Reverse_Deviation_Sort takes an extra input threshold, and ensures that only test patterns with output deviations not higher than threshold are selected (line 4). The parameter threshold is different for different circuits such that a reasonable number of test patterns are selected.

\section{EXPERIMENTAL RESULTS}

All experiments were performed on a Pentium 4 PC running Linux with a $2.6 \mathrm{Ghz}$ processor and $1 \mathrm{G}$ memory. The program to compute output deviations was implemented using $\mathrm{C}++$. It took approximately 80 seconds to compute the deviations for 10,000 vectors for a fullscan version of the largest benchmark circuit (s38584). Atalanta and its associated simulation engine are used to generate $n$-detection test sets and to perform fault simulation. The PROBE simulator [16] is used to compute the coverage of resistive bridging faults. We also implemented a bridging fault simulator to calculate the coverage of single non-feedback, zero-resistance bridging faults (sNFBFs). Due to the limitation of file size, for circuits c7552 and s9234 the set $T_{r e p}$ is a 7-detection test set; for other circuits, the set $T_{r e p}$ is a 15-detection test set.

To eliminate any bias in the comparison of different methods for test set selection, we use two arbitrarily chosen sets of confidence level vectors for our experiments. These vectors are defined separately for each gate type. For example, for a 2-input NAND gate, we use 1) "low confidence level" vector: $R^{N A N D 2}=$ $\left(0.8^{(00)}, 0.8^{(01)}, 0.8^{(10)}, 0.7^{(11)}\right)$, and 2) "high confidence level" vector: $R^{N A N D 2}=\left(0.95^{(00)}, 0.95^{(01)}, 0.95^{(10)}, 0.85^{(11)}\right)$. Since both sets of confidence level vectors yield similar results, we only report results obtained using the "high confidence level" set.

Gate exhaustive (GE) testing metrics are computed using an inhouse simulation tool based on the fault simulation program FSIM [24]. We use the three different GE metrics listed in Table II.

The metric score is the total number of distinct observed input combinations of all the gates, defined as $\sum_{i=1}^{N} \sum_{j=0}^{2^{n_{i}}-1} f_{i}(j)$, where $N$ is the total number of gates in the circuit, and $n_{i}$ is the number of inputs of gate $i$. An observed input combination of a gate is an innut combination that is applied to the gate inputs with the ga 


\begin{tabular}{c|l}
\hline Metric & Definition \\
\hline score & $\begin{array}{l}\text { Total number of all distinct observed input combinations } \\
\text { of all gates. }\end{array}$ \\
\hline exp-cnt & Number of gates that are exhaustively tested. \\
\hline ratio & $\begin{array}{l}\text { average observed input combinations ratio over all } \\
\text { gates. }\end{array}$ \\
\hline
\end{tabular}

TABLE II

GATE EXHAUSTIVE TESTING METRICS

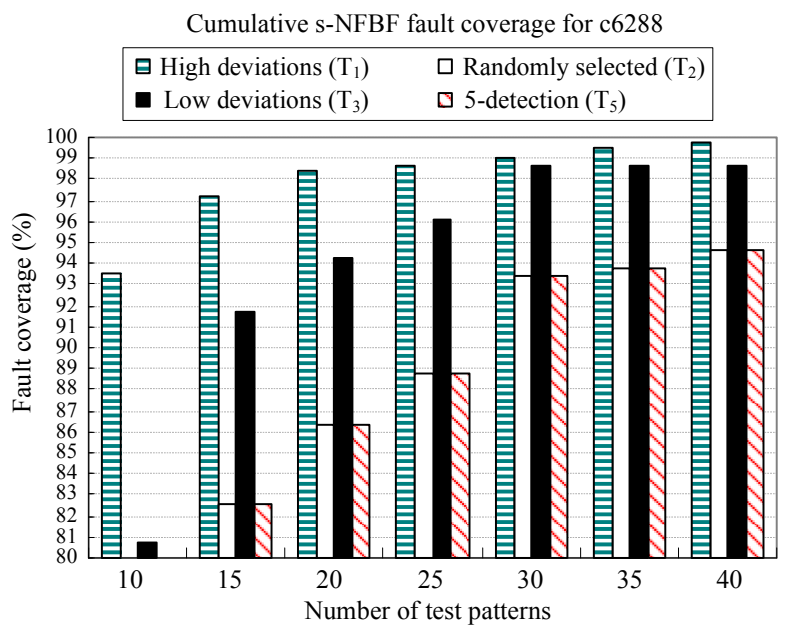

(a)

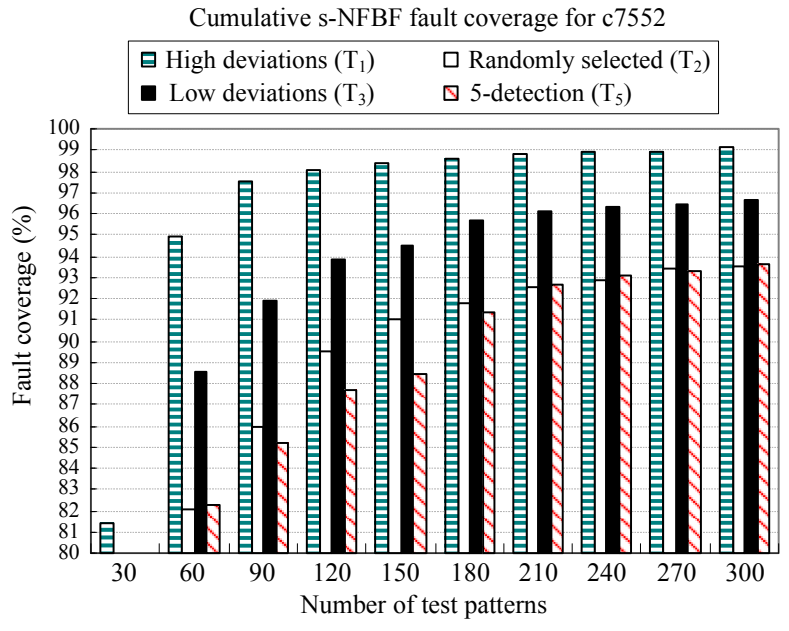

(b)

Fig. 6. Results for s-NFBF faults for two representative ISCAS-85 circuits.

being sensitized to at least one observation point such as a primary output or a scan cell. Function $f_{i}(j)$ is defined to be 1 if and only if the binary combination corresponding to the decimal number $j$ is applied to the inputs of gate $i$, and at the same time, the response of gate $i$ is observed at some observation points [17].

The metric exp-cnt simply counts the number of gates that are exhaustively tested. A gate is exhaustively tested if and only if all of its possible input combinations are applied and observed. The metric ratio is defined as $\sum_{i=1}^{N} \frac{\sum_{j=0}^{2^{n_{i}}-1} f_{i}(j)}{2^{n_{i}}-1} / N$; i.e., for each gate, we first calculate the ratio of observed input combinations to the number of all possible input combinations, and then obtain the average ratio over all gates.

We first present experimental results for the coverage obtained for s-NFBFs with the various test sets that we considered. We varied the test length to compare the effectiveness of the different tests. The first

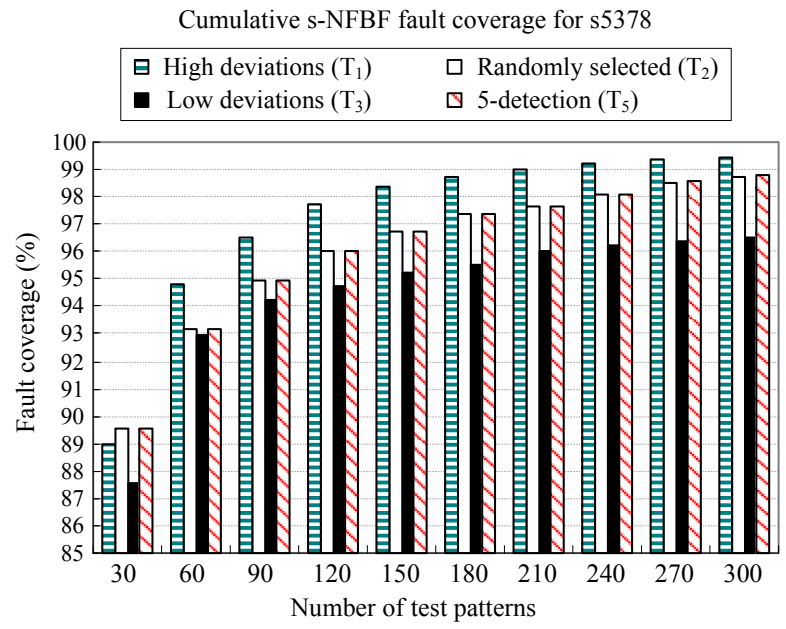

(a)

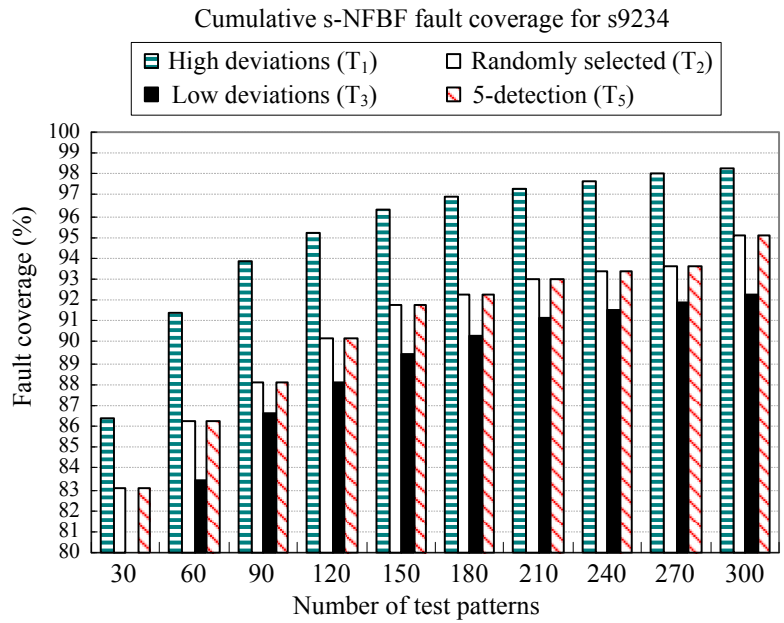

(b)

Fig. 7. Results for s-NFBF faults for two representative ISCAS-89 circuits.

set $T_{1}$ is determined using the procedure Deviation_Sort; it contains patterns with high deviations. Fault simulation is not necessary to obtain this test set. The second test set $T_{2}$ contains patterns selected at random from the base test set $T_{\text {rep }}$. The third test set $T_{3}$ is determined using the procedure Reverse_Deviation_Sort, i.e., it contains patterns with low deviations. The final test set is determined using fault simulation for 5-detection and therefore is named as $T_{5}$ instead of $T_{4}$. It is obtained from Procedure Subset by setting $n=5$.

Fig. 6-7 show that $T_{1}$ consistently provides higher fault coverage than the other test sets. The coverage for $T_{5}$ increases with test length, but it is considerably less than that for $T_{1}$ for shorter test lengths that may be desired for time-constrained production testing. Moreover, fault grading is required to generate $T_{5}$ from $T_{r e p}$, which may not be feasible in a production testing environment.

Next we present experimental results obtained for resistive bridging faults using the PROBE fault simulator. For these experiments, we again used the test sets $T_{1}, T_{3}$, and $T_{4}$. In addition, we generated another test set $T_{5}^{\star}$ using Method I described in Section III. Fault grading (based on resistive shorts) is combined with deviation-based ranking to generate this 5-detect test set, referred to as "Sorted 5detection".

Fig. 8 shows that $T_{1}$ and $T_{5}^{\star}$, the only two test sets that are obtained by selecting patterns with high deviations, consistently provi 


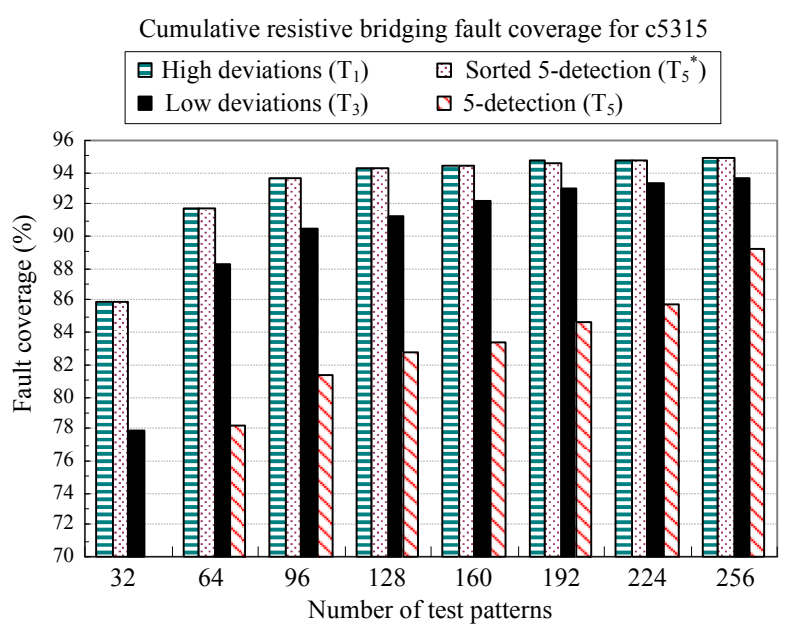

(a)
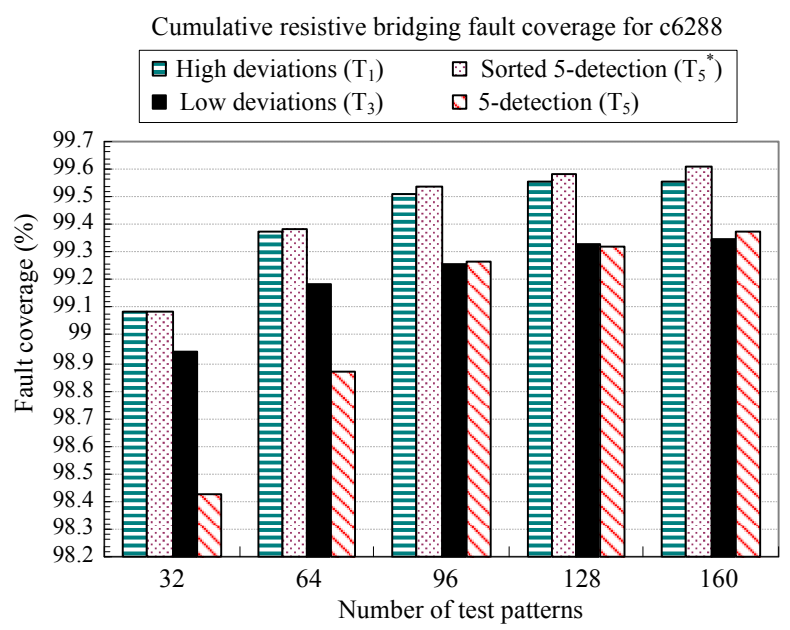

(b)

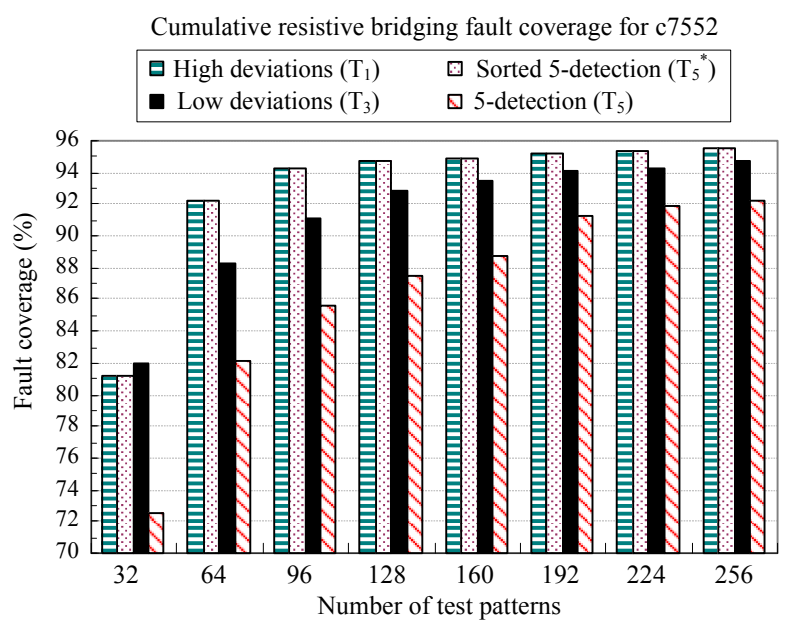

(c)

Fig. 8. Results for resistive bridging faults for the two largest ISCAS-85 circuits.

\begin{tabular}{|c|c|c|c|c|c|c|c|}
\hline \multirow{2}{*}{ Circuit } & \multirow{2}{*}{ Test set } & \multicolumn{6}{|c|}{$n$} \\
\cline { 3 - 8 } & $T_{n}$ & 900 & 1077 & 1235 & 1359 & 1472 & 1584 \\
\hline c5315 & $T_{n}^{\star}$ & 433 & 540 & 657 & 773 & 877 & 973 \\
\cline { 2 - 8 } & $T_{n}$ & 178 & 221 & 260 & 300 & 339 & 383 \\
\hline c6288 & $T_{n}^{\star}$ & 103 & 131 & 153 & 182 & 215 & 244 \\
\hline & $T_{n}$ & 1192 & 1491 & 1775 & 2032 & 2291 & - \\
\hline & $T_{n}^{\star} 7552$ & 799 & 1024 & 1244 & 1462 & 1664 & - \\
\hline
\end{tabular}

TABLE III

NUMBER OF PATTERNS IN $T_{n}^{\star}$ AND $T_{n}$ FOR DIFFERENT VALUES OF $n$.

\begin{tabular}{|c|c|c|c|c|c|}
\hline Circuit & Test set & \# patterns & score & exp-cnt & ratio \\
\hline c5315 & $T_{3}^{\star}$ & 433 & 9,388 & 1461 & $88.75 \%$ \\
& $T_{3}$ & 433 & 8,150 & 1155 & $78.04 \%$ \\
& $T_{5}^{\star}$ & 657 & 9,776 & 1474 & $88.87 \%$ \\
& $T_{5}$ & 657 & 8,903 & 1230 & $82.30 \%$ \\
\hline c6288 & $T_{3}^{\star}$ & 103 & 7,846 & 712 & $81.85 \%$ \\
& $T_{3}$ & 103 & 7,696 & 658 & $80.30 \%$ \\
& $T_{5}^{\star}$ & 153 & 7,857 & 723 & $81.96 \%$ \\
& $T_{5}$ & 153 & 7,729 & 679 & $80.64 \%$ \\
\hline c7552 & $T_{3}^{\star}$ & 799 & 11,759 & 1977 & $86.68 \%$ \\
& $T_{3}$ & 799 & 11,300 & 1851 & $84.79 \%$ \\
& $T_{5}^{\star}$ & 1244 & 11,814 & 1988 & $86.75 \%$ \\
& $T_{5}$ & 1244 & 11,502 & 1895 & $85.51 \%$ \\
\hline s5378 & $T_{3}^{\star}$ & 654 & 8,005 & 2263 & $92.78 \%$ \\
& $T_{3}$ & 654 & 7,949 & 2239 & $92.31 \%$ \\
& $T_{5}^{\star}$ & 1039 & 8,032 & 2276 & $92.93 \%$ \\
& $T_{5}$ & 1039 & 8,008 & 2264 & $92.79 \%$ \\
\hline s9234 & $T_{3}^{\star}$ & 907 & 14,234 & 4073 & $88.43 \%$ \\
& $T_{3}$ & 907 & 14,151 & 4064 & $88.16 \%$ \\
& $T_{5}^{\star}$ & 1372 & 14,269 & 4094 & $88.53 \%$ \\
& $T_{5}$ & 1372 & 14,235 & 4083 & $88.42 \%$ \\
\hline
\end{tabular}

TABLE IV

GATE EXHAUSTIVE TESTING RESULTS FOR SOME REPRESENTATIVE ISCAS BENCHMARK CIRCUITS.

fault coverage than the other test sets. In some cases the coverage of $T_{5}^{\star}$ is slightly higher than that of $T_{1}$, but in most cases the coverages of $T_{5}^{\star}$ and $T_{1}$ are the same. Therefore, in a production testing environment, $T_{1}$ may be a better choice than $T_{5}^{\star}$ since we do not need fault grading to generate $T_{1}$.

We next present the coverage for resistive bridging faults obtained using different $n$-detection test sets. For each value of $n$, we consider two test sets: the "Sorted $n$-detection" test set $\left(T_{n}^{\star}\right)$, obtained using output deviations and fault grading, and the baseline " $n$-detection" test set $\left(T_{n}\right)$, obtained using only fault grading. Since $T_{n}^{\star}$ always contains fewer test patterns than $T_{n}$, for the sake of fair comparison, $T_{n}$ is truncated to the size of $T_{n}^{\star}$. Table III lists the number of patterns in $T_{n}^{\star}$ and $T_{n}$ for different values of $n$. Fig. 9 shows that $T_{n}^{\star}$ consistently provides higher fault coverage than $T_{n}$.

Finally, we present results for the GE metrics obtained using deviation-sorted $n$-detect test sets $\left(T_{n}^{\star}\right)$ and $n$-detect test sets $\left(T_{n}\right)$ that do not use deviations ( $n=3$ or 5 ). $T_{n}$ is truncated to ensure a fair comparison for the same number of patterns. Table IV lists the results for some representative ISCAS benchmark circuits. As can been seen from Table IV, $T_{n}^{\star}$ provides higher values for all the three GE metrics.

\section{Conclusions}

We have shown that the output deviation is an effective metric to model the quality of test patterns. Pattern ranking based on output deviations offers a useful method for test-set selection during highvolume and time-constrained production testing. Experimental results show that for the same test length, test patterns selected using output deviations are consistently more effective than patterns selected using other methods. We have evaluated pattern grading using the fault coverage for resistive shorts and non-feedback bridging faults. and several gate exhaustive metrics. As part of ongoing work 


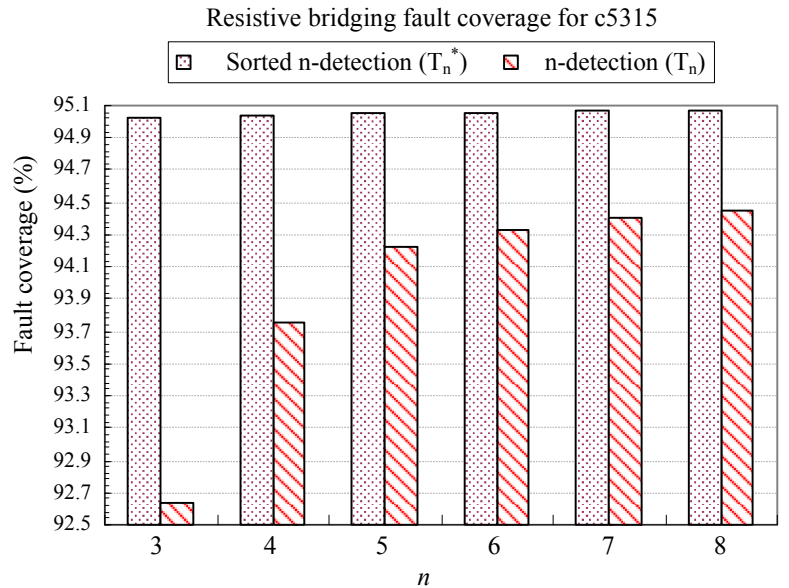

(a)

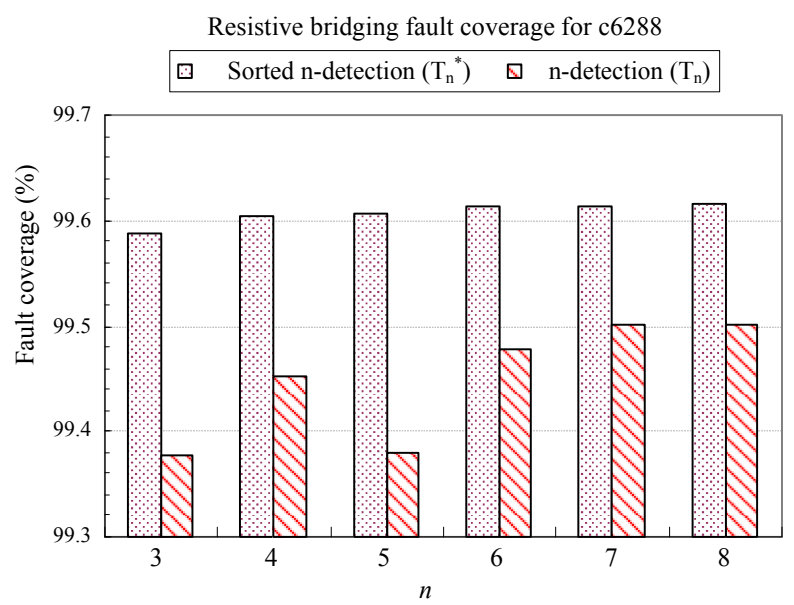

(b)

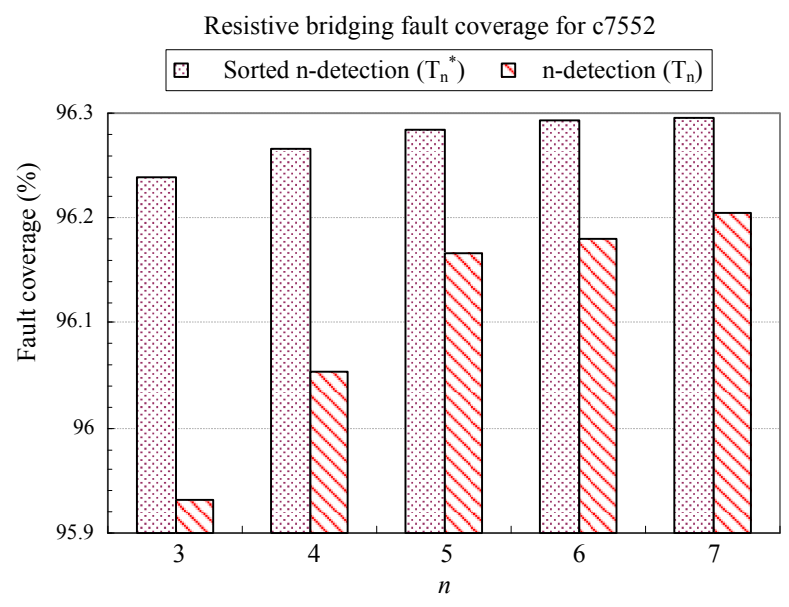

(c)

Fig. 9. Results for resistive bridging faults for the two largest ISCAS-85 circuits. comparing this method with other test pattern ranking techniques, such as a greedy algorithm which uses fault grading and ranks patterns based on the number of (modeled) faults detected with each pattern.

\section{ACKNOWLEDGMENT}

The authors thank Prof. Hank Walker of Texas A\&M University for providing the PROBE fault simulator.

\section{REFERENCES}

[1] J. Segura and C. F. Hawkins, CMOS Electronics : How It Works, How It Fails. Wiley-IEEE Press, 2004.

[2] R. Aitken, "New defect behavior at 130nm and beyond," in Informal Proc. European Test Symposium, 2004, pp. 279-284.

[3] F. Corno, P. Prinetto, M. Rebaudengo, and M. S. Reorda, "GATTO: a genetic algorithm for automatic test pattern generation for large synchronous sequential circuits," IEEE Trans. CAD, vol. 15, pp. 943951, Aug. 1996.

[4] P. Girard, C. Landrault, S. Pravossoudovitch, and B. Rodriguez, "A diagnostic ATPG for delay faults based on genetic algorithms," in Proc. IEEE Intl. Test Conf. on Test and Design Validity, 1996, pp. 286-293.

[5] Q. Xu and N. Nicolici, "Delay fault testing of core-based systems-ona-chip," in Proc. DATE Conf., 2003, pp. 744-749.

[6] I. Pomeranz and S. M. Reddy, "Pattern sensitivity: a property to guide test generation for combinational circuits," in Proc. Asian Test Symp., 1999 , pp. $75-80$.

[7] S. C. Ma, P. Franco, and E. J. McCluskey, "An experimental chip to evaluate test techniques experiment results," in Proc. ITC, 1995, pp. $663-672$.

[8] S. M. Reddy, I. Pomeranz, and S. Kajihara, "On the effects of test compaction on defect coverage," in Proc. VTS, 1996, pp. 430-435.

[9] I. Polian, I. Pomeranz, and B. Becker, "Exact computation of maximally dominating faults and its applications to n-detection tests," in Proc. Asian Test Symp., 2002, pp. 9-14.

[10] C. Tseng, S. Mitra, S. Davidson, and E. J. McCluskey, "An evaluation of pseudo random testing for detecting real defects," in Proc. VTS, 2001, pp. 404-409.

[11] S. Lee, B. Cobb, J. Dworak, M. R. Grimaila, and M. R. Mercer, "A new ATPG algorithm to limit test set size and achieve multiple detections of all faults," in Proc. DATE Conf., 2002, pp. 268-274.

[12] K. R. Kantipudi and V. D. Agrawal, "On the size and generation of minimal $n$-detection tests," in Proc. IEEE Intl. Conf. VLSI Design, 2006.

[13] Y. Tian, M. Mercer, W. Shi, and M. Grimaila, "An optimal test pattern selection method to improve the defect coverage," in Proc. ITC, 2005.

[14] S. Chakravarty and R. Galivanche, Intel Corporation, Private Communication.

[15] Z. Wang, K. Chakrabarty, and M. Goessel, "Test set enrichment using a probabilistic fault model and the theory of output deviations," in Proc. DATE Conf., 2006, pp. 1275-1280.

[16] C. Y. Lee and D. M. H. Walker, "PROBE: A PPSFP simulator for resistive bridging faults," in Proc. VTS, 2000, pp. 105-110.

[17] K. Y. Cho, S. Mitra, and E. J. McCluskey, "Gate exhaustive testing," in Proc. ITC, 2005, pp. 771-777.

[18] R. Guo, S. Mitra, E. Amyeen, J. Lee, S. Sivaraj, and S. Venkataraman, "Evaluation of test metrics: Stuck-at, bridge coverage estimate and gate exhaustive," in Proc. VTS, 2006.

[19] F. J. Ferguson and J. P. Shen, "A CMOS fault extractor for inductive fault analysis," IEEE Trans. CAD, vol. 7, pp. 1181-1194, Nov. 1988.

[20] K. P. Parker and E. J. McCluskey, "Probablistic treatment of general combinational networks," IEEE Trans. Computers, vol. C-24, pp. 668670, Jun. 1975.

[21] S. C. Seth, L. Pan, and V. D. Agrawal, "PREDICT-Probabilistic estimation of digital circuit testability," in Proc. Intl. Symp. Fault-Tolerant Computing, 1985, pp. 220-225.

[22] S. Krishnaswamy, I. L. Markov, and J. P. Hayes, "Logic circuit testing for transient faults," in Proc. European Test Symp., 2005, pp. 102-107.

[23] H. K. Lee and D. S. Ha, "On the generation of test patterns for combinational circuits," Dep't of Electrical Eng., Virginia Polytechnic Institute and State University, Tech. Rep. 12_93, 1993.

[24] — , "An efficient, forward fault simulation algorithm based on the parallel pattern single fault propagation," in Proc. ITC, 1991, pp. 946955. 